\title{
Fenol Çözeltisinin Farklı Elektrotlar Kullanılarak Elektrooksidasyonu
}

\author{
Orhan Taner CAN* \\ Bursa Teknik Üniversitesi, Çevre Mühendisliği Bölümü, Bursa \\ (ORCID: 0000-0003-3386-975X)
}

\begin{abstract}
$\ddot{\mathbf{O} z}$
$\mathrm{Bu}$ çalışmada toksik aromatik bir bileşik olarak bilinen fenolün BDD (Boron Doped Diamond), Ti/Pt ve MMO (Mixed Metal Oksit) elektrotları $\left(\mathrm{Ti} / \mathrm{RuO}_{2}-\mathrm{TiO}_{2}, \mathrm{Ti} / \mathrm{RuO}_{2}-\mathrm{IrO}_{2}, \mathrm{Ti} / \mathrm{IrO}_{2}-\mathrm{Ta}_{2} \mathrm{O}_{5}, \mathrm{Ti} / \mathrm{Pt}-\mathrm{IrO}_{2}\right)$ ile mineralizasyonu araştırıldı. Mineralizasyon seviyesinin belirlenmesi amacıyla deneylerin başında ve ilerleyen sürelerinde elektrooksidasyona tabi tutulan fenol çözeltisinden örnekler alınıp TOK (Toplam Organik Karbon) değerleri ölçüldü. Akım yoğunluğunun giderme verimine etkisini araştırmak için ise reaktöre üç farklı akım yoğunluğu (25 $\mathrm{mA} / \mathrm{cm}^{2}, 75 \mathrm{~mA} / \mathrm{cm}^{2}$ ve $125 \mathrm{~mA} / \mathrm{cm}^{2}$ ) uygulandı. Akım yoğunluğu artış1 ile mineralizasyon veriminin arttı̆̆ görüldü. $125 \mathrm{~mA} / \mathrm{cm}^{2}$ akım yoğunluğunda BDD anot 180. dakikadan itibaren \%100 giderme verimlerine ulaştı. Deneyin başında düşük performans gösteren Pt elektrotun 300 dakikalık deney süresi sonunda \% 84 lük giderme verimi ile, \% 72-79 aralığında verim gösteren MMO anotların performansını geçtiği belirlendi. Çalışmanın sonunda BDD elektrotun fenolün sulardan gideriminde diğer anotlardan daha iyi performans gösterdiği görüldü.
\end{abstract}

Anahtar kelimeler: Fenol, BDD, Pt, MMO elektrotlar, elektrooksidasyon.

\section{Electrooxidation of Phenol Solution Using Several Electrodes}

\begin{abstract}
In this study, mineralization of phenol, known as toxic aromatic compound, was investigated using BDD (Boron Doped Diamond), Ti/Pt and MMO (Mixed Metal Oxide) electrodes ( $\mathrm{Ti} / \mathrm{RuO}_{2}-\mathrm{TiO}_{2}, \mathrm{Ti} / \mathrm{RuO}_{2}-\mathrm{IrO}_{2}, \mathrm{Ti}_{\mathrm{IrO}}-\mathrm{Ta}_{2} \mathrm{O}_{5}$, $\mathrm{Ti} / \mathrm{Pt}-\mathrm{IrO}_{2}$ ). In order to determine the level of mineralization, samples were taken from the phenol solution subjected to electrooxidation at the beginning and at the during of the experiments and TOC (Total Organic Carbon) values were measured. Three different current densities $\left(25 \mathrm{~mA} / \mathrm{cm}^{2}, 75 \mathrm{~mA} / \mathrm{cm}^{2}\right.$ and $\left.125 \mathrm{~mA} / \mathrm{cm}^{2}\right)$ were applied to the reactor to investigate the effect of current density on the removal efficiency. BDD anode reached $100 \%$ removal efficiency from 180 minutes at $125 \mathrm{~mA} / \mathrm{cm}^{2}$ current density. At the beginning of the experiment, the low performance Pt electrode showed $84-\%$ removal efficiency at the end of the 300 minutes test period. MMO anodes exceeding the yield range. At the end of the study, BDD electrode performed better than other anodes in the removal of phenol from water.
\end{abstract}

Keywords: Phenol, BDD, Pt, MMO electrodes, electrooxidation.

\section{Giriş}

Fenoller, bir veya daha fazla hidroksil grubunun aromatik halkaya bağlı olduğu aromatik kalıcı organik bileşiklerdir. Yemek yapımı, yıkama ve banyo gibi evsel faaliyetlerin yanı sıra petrol rafinerileri, çelik fabrikaları, boya üretimi, kimyasal sentez, kağıt, tekstil, deterjan, ilaç ve plastik endüstrilerinin atıksularında bulunurlar [1-6]. Fenolün endüstriyel atıklardan uzaklaştırılması çevre ve insan sağlığı için gereklidir. Fenol balığın tadı ve kokusunda değişikliğe sebep olurken ve bakteri, alg, memeli ve insan üzerinde zararlı etkilere neden olur [7]. Geleneksel arıtma yöntemleri fenol gideriminde etkili olmakla beraber, solvent ekstraksiyonu ve aktif karbon adsorbsiyonu gibi proseslerde yüksek maliyet vb sebeplerle ekonomik olmamaktadır. Biyolojik arıtma yöntemlerinde ise bakteriyel inhibisyona neden

\footnotetext{
*Sorumlu yazar: orhan.can@btu.edu.tr

Geliş Tarihi: 22.09.2019, Kabul Tarihi: 11.02.2020
} 
olmaktadır [8,9]. Bu yöntemlerin dişında, fotokatalitik bozunma [6, 10, 11], peroksit / UV [12], süperkritik su oksidasyonu [13] ve sonokimyasal bozunma [14] fenolün sulardan gideriminde kullanılmıştır. Elektrokimyasal arıtma toksik organik atıkları parçalamak için alternatif bir yöntemdir. Üstelik elektroliz, biyolojik arıtmanın detoksifikasyonunda ön işlem teknolojisi olarak kullanılabilir [14]. Elektrokimyasal yöntem çevre dostu ve umut verici bir yöntem olarak, uyumluluk, enerji verimliliği, düşük hacimli uygulama, çok yönlülük ve otomasyona uygunluk açısından bazı avantajlar sunar. Bu çalışmanın amacı fenol çözeltisinin elektrooksidasyon ile mineralizasyonunda farklı elektrot tiplerinin ve akım yoğunluğunun proses verimine etkisinin incelenmesidir. Bu amaçla BDD, Pt ve MMO elektrotlardan oluşan bir dizi farklı anot kullanılarak farklı akım yoğunluklarında fenolün mineralizasyon verimleri TOC cinsinden ölçülerek proses verimleri araştırıldı.

\section{Materyal ve Metot}

\subsection{Materyal}

Deneylerde Merck marka fenol kullanıldı. Fenol $\mathrm{C}_{6} \mathrm{H}_{5} \mathrm{OH}$ kimyasal formüle sahip olup, molar kütlesi $94.11 \mathrm{~g} / \mathrm{mol}$ dür. Fenola ait kimyasal yapı Şekil 1'de görülmektedir.

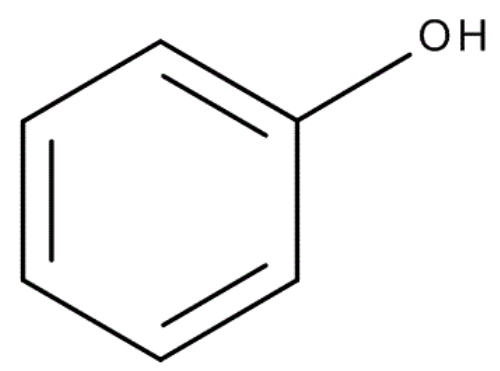

Şekil 1. Yapısal formül görüntüsü

\subsection{Metot}

Deneylerde Şekil 2'de şematik olarak gösterilen deney düzeneği kullanılmıştır. Her çalışma için pyrex cam reaktöre $500 \mathrm{ml}$ fenol çözeltisi konuldu. Elektrotlar arası iletkenlik sağlamak için, çözeltiye elektrolit olarak $\mathrm{NaCl}$ eklenmiştir. Reaktörde, bir anot ve bir katottan oluşan $200 \times 60 \times 2 \mathrm{~mm}$ boyutlarında iki elektrot kullanıldı. BDD (Boron Doped Diamond), Ti/Pt ve MMO (Mixed Metal Oksit) elektrotlar1 $\left(\mathrm{Ti} / \mathrm{RuO}_{2}-\mathrm{TiO}_{2}, \mathrm{Ti} / \mathrm{RuO}_{2}-\mathrm{IrO}_{2}, \mathrm{Ti} / \mathrm{IrO}_{2}-\mathrm{Ta}_{2} \mathrm{O}_{5}, \mathrm{Ti} / \mathrm{Pt}-\mathrm{IrO}_{2}\right)$ anot olarak kullanılırken katot olarak paslanmaz çelik bir elektrot kullanıldı. Çözeltinin daldırma seviyesine bağlı olarak anotların toplam aktif yüzey alanı, $40 \mathrm{~cm}^{2}$ olarak hesaplandı. Her çalıştırmadan önce, elektrotlar, yüzeyde biriken safsızlıkları gidermek için aseton veya fosforik asit ile silindi ve damıtılmış suyla yıkandı. Sistemde Agilent ve Rigol marka programlanabilir (Agilent U8032A 0-60V / 0-3A Agilent U8002A 0-30V / 05A Rigol DP832 0-30V / 0-3A) dijital doğru akım (DC) güç kaynakları kullanılmıştır. Deney süresi boyunca sıcaklıktaki artış, reaktörün dış duvarına hava verilerek $25-35^{\circ} \mathrm{C}$ 'de tutuldu. Tüm kimyasal analizler, su ve atık suyun incelenmesi için standart yöntemler izlenerek gerçekleştirildi. TOK konsantrasyonlarını tahmin etmek için TOK cihazı (Teledyne Tekmar marka, Torch model) kullanıldı. TOK giderme verimi, $E$, aşağıdaki gibi hesaplandı

$$
E(\%)=\frac{C_{i}-C_{f}}{C_{i}} \times 100
$$

Burada; $C_{i}$ başlangıç TOK konsantrasyonu (mg/L), $C_{f}$ ise son TOK konsantrasyonu (mg/L).

Elektrokimyasal arıtmanın temel maliyet bileşeni olarak değerlendirilen spesifik enerji tüketimi (specific energy consumption, SEC) TOK giderme verimine bağlı olarak hesaplandı [15].

$$
S E C=(V * I * t) / T O K_{\text {rem }}
$$


Burada; V reaktördeki voltaj, I amper cinsinden akım, t saat cinsinden süre ve $\mathrm{TOK}_{\text {rem }}$ giderilen TOK değeridir.

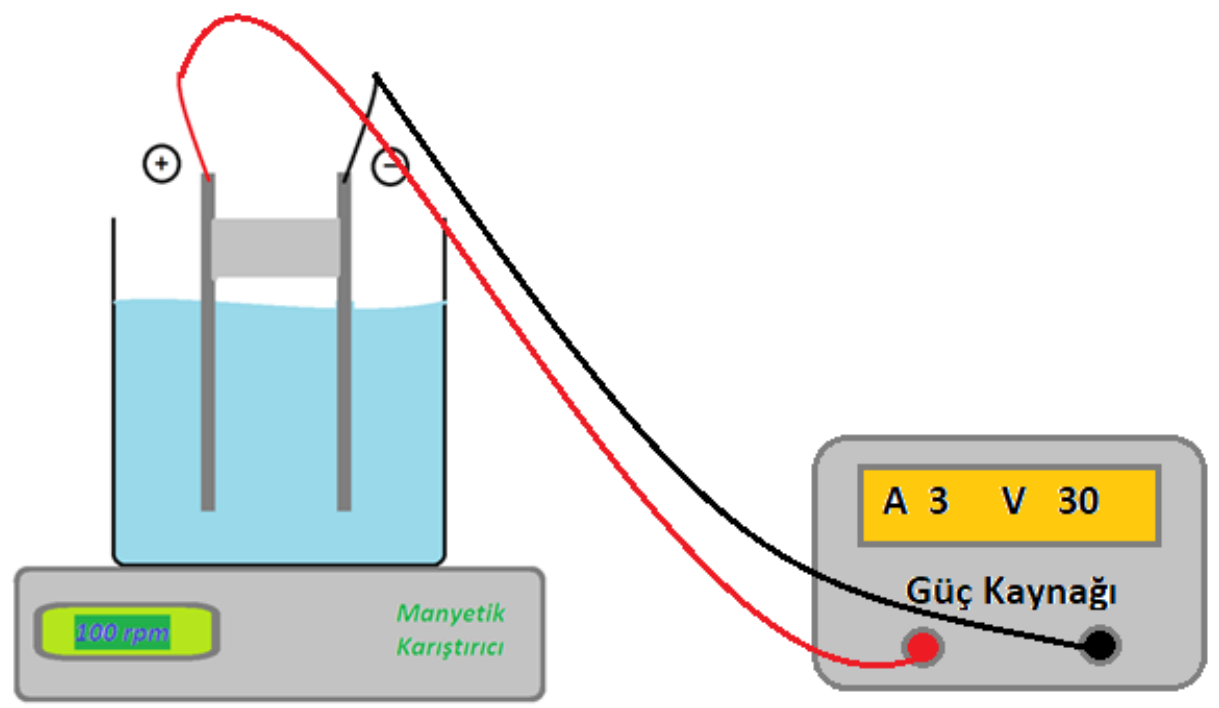

Şekil 2. Deney düzeneğinin şematik gösterimi

\section{Bulgular ve Tartışma}

Fenol çözeltisinden TOK gideriminde anot elektrot etkisini araştırmak için altı farklı tipte anot kullanıldı. Akım yoğunluğunun giderme verimine etkisini araștırmak için ise reaktöre üç farklı akım yoğunluğu $\left(25 \mathrm{~mA} / \mathrm{cm}^{2}, 75 \mathrm{~mA} / \mathrm{cm}^{2}\right.$ ve $\left.125 \mathrm{~mA} / \mathrm{cm}^{2}\right)$ uygulandı. Deneyler 300 dakikalık çalışma süresinde tamamlandı ve saat başı reaktörden örnek alınıp TOK analizi yapıldı. Ayrıca, TOK giderimi için tüketilen elektrik miktarına bağlı olarak SEC hesaplandı.

Şekil 3'de farklı anotların farklı akım yoğunluğundaki TOK giderme verimleri ve SEC sonuçları görülmektedir. Şekil 1 (a)'da görüleceği üzere $25 \mathrm{~mA} / \mathrm{cm}^{2}$ akım yoğunluğunda BDD anot ile $\mathrm{Pt}$ ve MMO anotlardan daha iyi giderme verimi elde edildi ve 300 dakikalık deney süresi sonunda BDD anot ile \% 81 lik bir verime ulaşıldı. Bununla birlikte Pt elektrotla \% 39 ile en kötü giderme verimine ulaşıldı. MMO anotlarla ise \% 45-65 aralığında değișen giderme verimlerine ulașıldı. Șekil 3 (c)'de ise akım yoğunluğunun $75 \mathrm{~mA} / \mathrm{cm}^{2}$, ye çıkarılmasıyla giderme verimlerinin tüm anot elektrotlar için arttığ1 görülmektedir. $\mathrm{Bu}$ akım yoğunluğunda BDD anodun 300 dakikalık deney süresi sonunda çözeltideki fenolün tamamını mineralize etme başarısı gösterdiği belirlendi. Pt anot ise çalışmanın ilk saatinde diğer anotlara göre geride kalmasına rağmen zamanla performansının arttığ 1 ve 300 dakikalık deney süresi sonunda \% 68 lik giderme verimi ile, \% 61-70 aralığında verim gösteren MMO anotların performansına yetiştiği belirlendi. $125 \mathrm{~mA} / \mathrm{cm}^{2}$ akım yoğunluğunda (Şekil 3 (e)) ise BDD anodun 180. dakikadan itibaren \%100 giderme verimlerine ulaştığ , deneyin başında düşük performans gösteren Pt elektrotun 300 dakikalık deney süresi sonunda \% 84 lük giderme verimi ile, \% 72-79 aralığında verim gösteren MMO anotların performansını geçtiği belirlendi. Duan ve arkadaşlarının $\mathrm{PbO}_{2}$ anot kullanarak $50 \mathrm{mg} / \mathrm{L}$ giriş konsantrasyonuna sahip fenol çözeltisine 180 dakika boyunca $20 \mathrm{~mA} / \mathrm{cm}^{2}$ akım yoğunluğu uygulayarak \%86 lik TOC giderimi elde etmişlerdir [16]. Bir başka çalışmada Song ve arkadaşlarının $\mathrm{PbO}_{2}$-Sn anot kullanarak $100 \mathrm{mg} / \mathrm{L}$ giriş konsantrasyonuna sahip fenol çözeltisine 480 dakika boyunca $10 \mathrm{~mA} / \mathrm{cm}^{2}$ akım yoğunluğu uygulayarak \%49 luk TOC giderimi elde etmişlerdir [17]. Canizares ve arkadaşları ise BDD anot kullanarak $360-1450 \mathrm{mg} / \mathrm{L}$ TOC giriş konsantrasyonuna sahip fenol çözeltisine 180 dakika boyunca $15-60 \mathrm{~mA} / \mathrm{cm}^{2}$ akım yoğunluğu uygulayarak TOC nin neredeyse tamamını gidermeyi başarmışlardır [18]. 

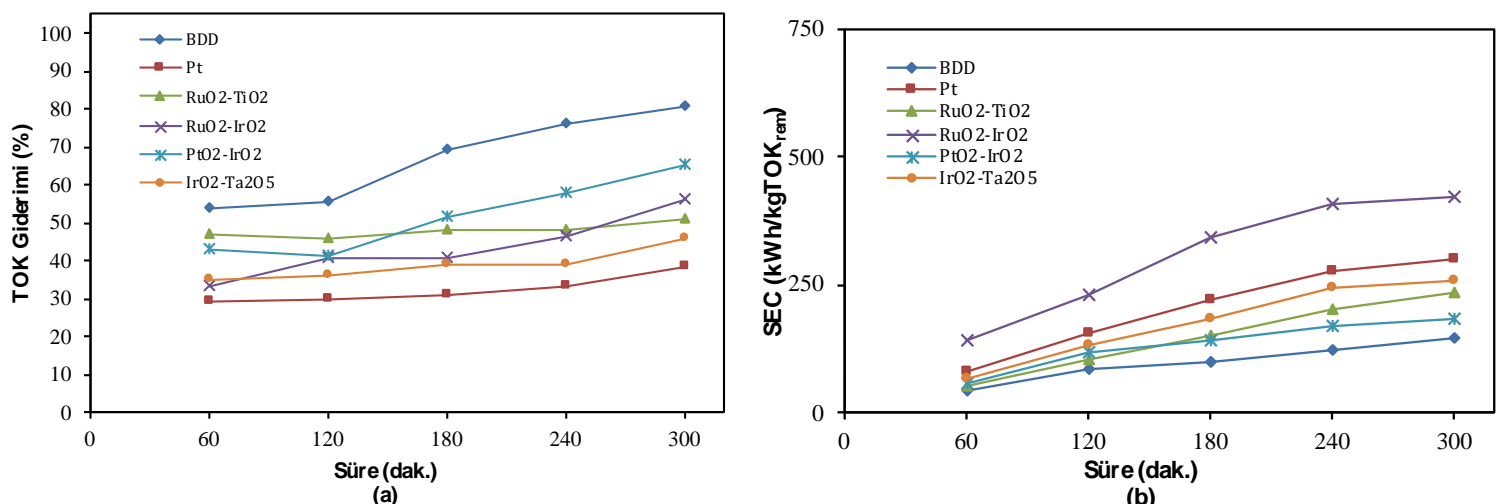

(b)
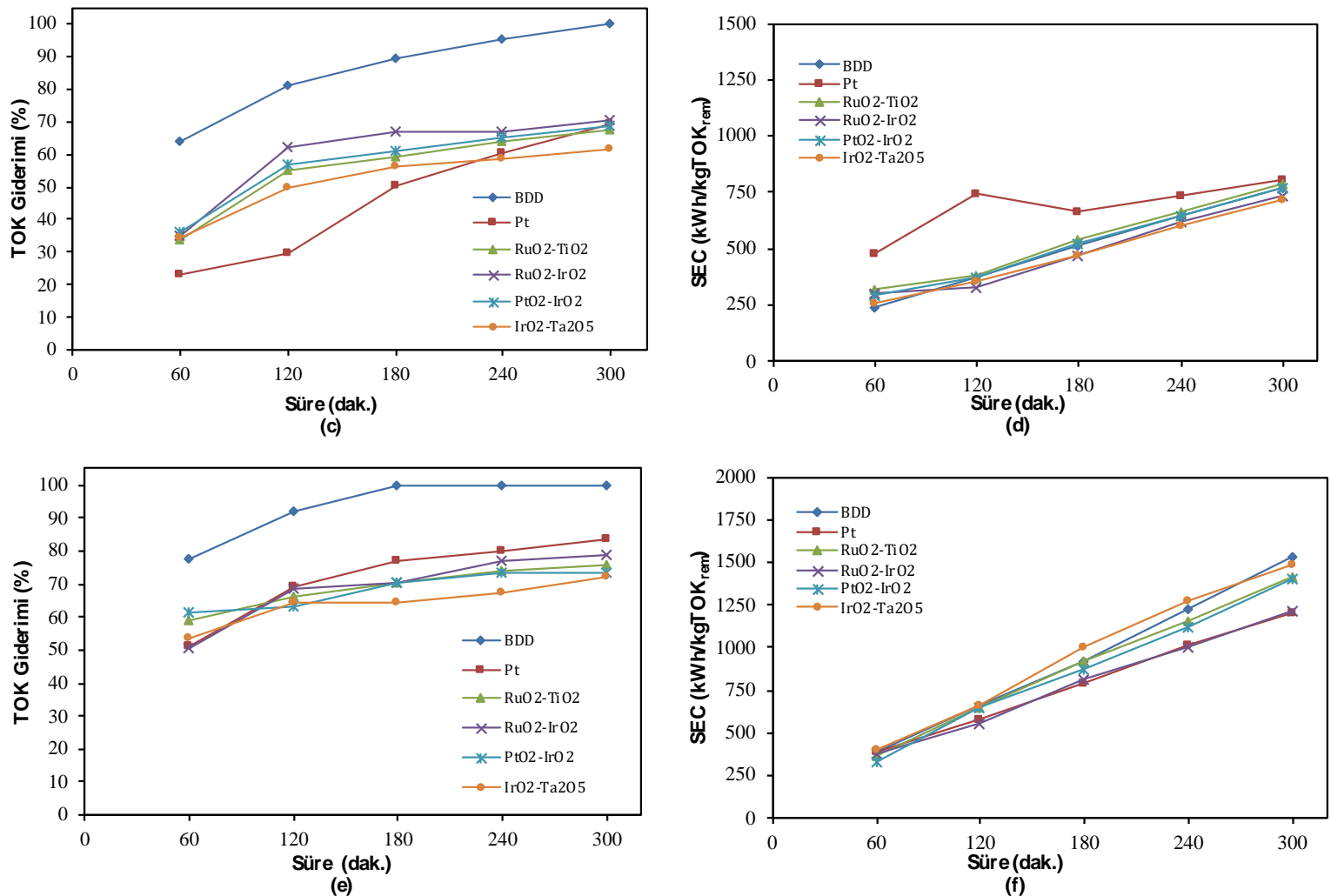

Şekil 3. BDD, Pt ve MMO elektrotların (a) $25 \mathrm{~mA} / \mathrm{cm}^{2}$ akım yoğunluğunda giderme verimine (b) SEC üzerine etkisi, (c) $75 \mathrm{~mA} / \mathrm{cm}^{2}$ akım yoğunluğunda giderme verimine (d) SEC üzerine etkisi, (e) $125 \mathrm{~mA} / \mathrm{cm}^{2} \mathrm{akım}$ yoğunluğunda giderme verimine (f) SEC üzerine etkisi. (deney şartları: Fenol $\mathrm{C}_{0}$ : $0.5 \mathrm{ml} / \mathrm{L}$, karıştırma hızı: 500 rpm, elektrotlar arası mesafe: 15 mm, elektrolit konsantrasyonu: $\mathrm{NaCl} 6000 \mathrm{mg} / \mathrm{L}$ ).

BDD, potansiyel olarak aşırı oksijen oluşumu, düşük kapasitans ve elektrokimyasal kararlılı̆̆1 nedeniyle organik kirleticilerin oksidasyonunda bilinen tüm elektrot malzemelerinin en iyi elektrotudur [19]. BDD elektrot fenolik bileşikler gibi kirleticilerin gideriminde ise oldukça etkili olduğu rapor edilmiştir [20]. Bununla birlikte, BDD elektrotun dezavantajlı tarafi endüstriyel atık su arıtımında maliyetli olmasıdır [21]. Diğer taraftan MMO elektrotların, özellikle klor-alkali prosesi için, geniş endüstriyel uygulamaya sahip olduğu ve yüksek akım yoğunlukları koşullarında bile nispeten ucuz ve çok kararlı olduğu bilinmektedir [22].

Şekil 3 (b), (d) ve (f) de ise sırasıyla 25, 75 ve $125 \mathrm{~mA} / \mathrm{cm}^{2}$ akım yoğunluklarında her bir anodun TOK giderme verimlerinden hesaplanan SEC miktarları görülmektedir. SEC miktarının uygulanan akım yoğunluğu ile arttığı belirlendi. Her ne kadar akım yoğunluğu artırıldığında giderme verimleri arttı ise, giderme verimlerinin harcanan enerji ile orantılı olarak artmamasından dolayı SEC giderme verimleri işletme maliyeti açısından akım yoğunluğunu arttırmanın olumsuz olduğunu göstermektedir. Yüksek maliyetlerine rağmen BDD elektrot ile oksidasyonda özellikle düşük akım yoğunluğunda diğer 
anotlardan daha düşük SEC değerleri elde edilmektedir. Bu da işletme maliyetinde enerji harcamaları açısından BDD elektrotları avantajlı duruma getirmektedir.

Elektrokimyasal mineralizasyonda, oksijen elektrik enerjisi kullanılarak sudan organik kirleticiye aktarılır. Bu elektrokimyasal oksijen transfer reaksiyonu olarak adlandırılır (EOTR, Electrochemical Oxygen Transfer Reaction). Aşağıda fenolün anodik mineralizasyonu gösterildi (eşitlik 3) [23].

$$
\mathrm{C}_{6} \mathrm{H}_{5} \mathrm{OH}+11 \mathrm{H}_{2} \mathrm{O} \rightarrow 6 \mathrm{CO}_{2}+28 \mathrm{H}^{+}+28 e^{-}
$$

Elektrot materyalinin yapısı oksidasyon prosesini güçlü bir şekilde etkilediği bilinmektedir [22, 24]. Elektroksidasyon sırasında su molekülünden $\mathrm{OH}$ radikali üretilmesi işleminde üretilen $\mathrm{OH}$ radikalinin elektrot yüzeyinde tutunma şekline göre elektrotlar aktif ve aktif olmayan anot olmak üzere iki gruba ayrılırlar. OH radikalini kimyasal olarak bağlayan grafit, $\mathrm{IrO} 2, \mathrm{RuO} 2$ ve platin gibi elektrotlar aktif elektrotlar olarak adlandırılırken, fiziksel olarak bağlayan kurşun oksit ve BDD gibi elektrotlar aktif olmayan elektrotlar olarak adlandırılmaktadırlar [22, 24]. Bu çalışmada kullanılan MMO elektrotlar aktif elektrotlar özelliklerini göstermiş ve $\mathrm{OH}$ radikalinin fenol gideriminde etkinliği, $\mathrm{OH}$ radikalinin fiziksel olarak bağlı olduğu BDD elektrottan daha düşük seviyededir.

\section{Sonuç ve Öneriler}

Toksik aromatik bir bileşik olarak bilinen fenolün sulardan gideriminde farklı elektrot materyallerinin veriminin araştırıldığı bu çalışmanın sonucunda, fenolün mineralizasyonu başarı ile sağlandı. TOK giderme verimi sonuçlarından elektrot türünün giderme verimi üzerinde önemli bir etkisinin olduğu görüldü. Benzer şekilde çözeltiye uygulanan akım yoğunluğundaki artışın fenolün mineralizasyonunda etkisinin büyük olduğu görüldü. Daha önceki çalışmaların sonuçlarına benzer şekilde BDD elektrotun Pt ve MMO elektrotlardan daha iyi mineralizasyon değerlerine ulaştığ 1 tespit edildi. Yüksek maliyetlerine rağmen BDD elektrotlar organik kirleticilerin sulardan gideriminde ideal anotlar olarak değerlendirilmektedir.

\section{Yazarların Katkısı}

Makalede tüm katkı şahsıma aittir.

\section{Çıkar Çatışması Beyanı}

Yazarlar arasında herhangi bir çıkar çatışması bulunmamaktadır.

\section{Araştırma ve Yayın Etiği Beyanı}

Yapılan çalışmada araştırma ve yayın etiğine uyulmuştur.

\section{Kaynaklar}

[1] Iniesta J., González-García J., Expósito E., Montiel V., Aldaz A. 2001. Influence of chloride ion on electrochemical degradation of phenol in alkaline medium using bismuth doped and pure $\mathrm{PbO} 2$ anodes. Water Research, 35 (14): 3291-3300.

[2] Awad Y.M., Abuzaid N.S. 2000. The influence of residence time on the anodic oxidation of phenol. Separation and Purification Technology, 18 (3): 227-236.

[3] Li X., Cui Y., Feng Y., Xie Z., Gu J.-D. 2005. Reaction pathways and mechanisms of the electrochemical degradation of phenol on different electrodes. Water Research, 39 (10): 19721981.

[4] Yavuz Y., Koparal A.S. 2006. Electrochemical oxidation of phenol in a parallel plate reactor using ruthenium mixed metal oxide electrode. Journal of Hazardous Materials, 136 (2): 296-302.

[5] Yang X., Zou R., Huo F., Cai D., Xiao D. 2009. Preparation and characterization of Ti/SnO2- 
$\mathrm{Sb} 2 \mathrm{O} 3-\mathrm{Nb} 2 \mathrm{O} 5 / \mathrm{PbO} 2$ thin film as electrode material for the degradation of phenol. Journal of Hazardous Materials, 164 (1): 367-373.

[6] Li M., Feng C., Hu W., Zhang Z., Sugiura N. 2009. Electrochemical degradation of phenol using electrodes of Ti/RuO2-Pt and Ti/IrO2-Pt. Journal of Hazardous Materials, 162 (1): 455-462.

[7] Verschueren K. 1977. Handbook of Environmental Data on Organic Chemicals. Wiley.

[8] Abuzaid N.S., Nakhla G.F. 1996. Effect of solution pH on the kinetics of phenolics uptake on granular activated carbon. Journal of Hazardous Materials, 49 (2): 217-230.

[9] Nakhla G.F., Al-Harazin I.M. 1993. Simplified analysis of biodegradation kinetics of phenolic compounds by heterogeneous cultures. Environmental Technology, 14 (8): 751-760.

[10] Chiou C.-H., Juang R.-S. 2007 Photocatalytic degradation of phenol in aqueous solutions by Prdoped TiO2 nanoparticles. Journal of Hazardous Materials, 149 (1): 1-7.

[11] Adán C., Bahamonde A., Fernández-García M., Martínez-Arias A. 2007. Structure and activity of nanosized iron-doped anatase $\mathrm{TiO} 2$ catalysts for phenol photocatalytic degradation. Applied Catalysis B: Environmental, 72 (1): 11-17.

[12] Barakat M.A., Tseng J.M., Huang C.P. 2005. Hydrogen peroxide-assisted photocatalytic oxidation of phenolic compounds. Applied Catalysis B: Environmental, 59 (1): 99-104.

[13] Matsumura Y., Nunoura T., Urase T., Yamamoto K. 2000. Supercritical water oxidation of high concentrations of phenol," Journal of Hazardous Materials, 73 (3): 245-254.

[14] Nakui H., Okitsu K., Maeda Y., Nishimura R. 2007. Effect of coal ash on sonochemical degradation of phenol in water. Ultrasonics Sonochemistry, 14 (2): 191-196.

[15] Can O.T. 2015. Removal of TOC from fertilizer production wastewater by electrooxidation. Desalination and Water Treatment, 53 (4).

[16] Duan X., Ma F., Yuan Z., Chang L., Jin X. 2013. Electrochemical degradation of phenol in aqueous solution using $\mathrm{PbO} 2$ anode. Journal of the Taiwan Institute of Chemical Engineers, 44 (1): 95-102.

[17] Song S., Zhan L., He Z., Lin L., Tu J., Zhang Z., Chen J. 2010. Mechanism of the anodic oxidation of 4-chloro-3-methyl phenol in aqueous solution using $\mathrm{Ti} / \mathrm{SnO} 2-\mathrm{Sb} / \mathrm{PbO} 2$ electrodes. Journal of Hazardous Materials, 175 (1): 614-621.

[18] Cañizares P., Díaz M., Domínguez J.A., García-Gómez J., Rodrigo M.A. 2002. Electrochemical Oxidation of Aqueous Phenol Wastes on Synthetic Diamond Thin-Film Electrodes. Industrial \& Engineering Chemistry Research, 41 (17): 4187-4194.

[19] Martínez-Huitle C.A., Brillas E. 2009. Decontamination of wastewaters containing synthetic organic dyes by electrochemical methods: A general review. Applied Catalysis B: Environmental, 87 (3): 105-145.

[20] Polcaro A.M., Vacca A., Palmas S., Mascia M. 2003. Electrochemical treatment of wastewater containing phenolic compounds: oxidation at boron-doped diamond electrodes. Journal of Applied Electrochemistry, 33 (10): 885-892.

[21] Zhou M., Särkkä H., Sillanpää M. 2011. A comparative experimental study on methyl orange degradation by electrochemical oxidation on BDD and MMO electrodes. Separation and Purification Technology, 78 (3): 290-297.

[22] Simond O., Schaller V., Comninellis C. 1997. Theoretical model for the anodic oxidation of organics on metal oxide electrodes. Electrochimica Acta, 42 (13): 2009-2012.

[23] Panizza M., Cerisola G. 2009. Direct And Mediated Anodic Oxidation of Organic Pollutants. Chemical Reviews, 109 (12): 6541-6569.

[24] Comninellis C. 1994. Electrocatalysis in the electrochemical conversion/combustion of organic pollutants for waste water treatment. Electrochimica Acta, 39 (11): 1857-1862. 